\title{
Use of artificial neural networks in the management of antenatally diagnosed ureteropelvic junction obstruction
}

\author{
Ilker Seçkiner, MD; Serap Ulusam Seçkiner, PhD; Ömer Bayrak, MD; Sakıp Erturhan, MD*
}

Cite as: Can Urol Assoc J 2011;5(6):E152-E155; http://dx.doi.org/10.5489/cuaj.10043. Epub 2011 Mar 1.

\begin{abstract}
Background: In this study, an artificial neural network (ANN) based system has been developed specifically to help in the management of antenatally diagnosed uretero-pelvic junction (UPJ) obstruction. Methods: A total of 53 infants with antenatally detected hydronephrosis caused by UPJ obstruction were included in this study. A neural network was developed with the help of a commercially available software package. The patients' age and sex, renal pelvic diameter, laterality, split renal function and presence of renal scar on radionuclide scan, follow-up times, urine culture results and the presence of symptomatic infections were used as variables. These data were also entered into a statistical software package and linear regression analysis was done.

Results: During the follow-up period, 36 children were observed, and the remaining 17 renal units underwent pyeloplasty. The average sensitivity of the ANN model in predicting the outcome was found to be $92 \%$ in the training group and $75 \%$ in the validation and test groups. In linear regression, none of the predictors were found to be statistically significant.

Interpretation: In this study, we have demonstrated that the use of ANNs in antenatally diagnosed UPJ obstruction can help the clinician in making treatment decisions, and thus can be useful in daily clinical practice.
\end{abstract}

\section{Résumé}

Contexte : Dans cette étude, un système fondé sur un réseau de neurones artificiels a été mis au point précisément pour aider à la prise en charge d'une obstruction de la jonction urétéropelvienne diagnostiquée pendant la période anténatale.

Méthodologie : Au total, 53 enfants atteints d'hydronéphrose décelée avant la naissance et causée par l'obstruction de la jonction urétéropelvienne ont été inclus dans cette étude. Un réseau de neurones a été élaboré à l'aide d'un logiciel offert sur le marché. L'âge et le sexe du patient, le diamètre du bassinet du rein, la latéralité, une fonction rénale séparée et la présence de cicatrices rénales observables par scintigraphie par balayage, la durée de la période de suivi, les résultats d'analyses d'urine et la présence d'infections symptomatiques ont été utilisés comme variables. Ces données ont également été saisies dans un progiciel statistique et ont servi à une analyse de régression linéaire.
Résultats : Au cours de la période de suivi, 36 enfants ont été placés en observation, et on a procédé à une pyéloplastie dans les 17 autres cas. La sensibilité moyenne du modèle pour la prédiction de l'issue a été évaluée à $92 \%$ dans le groupe de formation et à $75 \%$ dans les groupes de validation et de test. Dans I'analyse de régression linéaire, aucun des facteurs de prédiction n'a été jugé significatif sur le plan statistique.

Interprétation : Dans cette étude, nous avons montré que l'utilisation d'un réseau de neurones artificiels en présence d'obstruction de la jonction urétéropelvienne diagnostiquée avant la naissance peut aider le clinicien à prendre des décisions thérapeutiques, et peut donc être utile dans la pratique clinique quotidienne.

\section{Introduction}

Fetal hydronephrosis is the most common anomaly detected on antenatal ultrasound examination and affects $1 \%$ to $5 \%$ of pregnancies. ${ }^{1}$ Despite its frequency, however, the appropriate postnatal management of prenatal hydronephrosis has not been well-defined. ${ }^{2}$

Ureteropelvic junction (UPJ) obstruction is the most common pathologic cause of antenatally detected hydronephrosis. The therapeutic options for antenatally diagnosed UPJ obstruction are observation and surgery. The appropriate patients for observation are those with significant hydronephrosis on sonogram in whom the renal scan demonstrates $>40 \%$ of split renal function from the apparently obstructed kidney, even if washout is delayed. ${ }^{3,4}$ The surgical approach favoured in the repair of UPJ obstruction is dismembered pyeloplasty; this approach has been shown to be effective due to its broad applicability, means of allowing excision of the pathologic segment in question and facilitation in reducing pyeloplasty when necessary. ${ }^{5}$

Artificial neural networks (ANN) are computational methodologies that perform multifactorial analyses; they are inspired by networks of biological neurons. These networks can be trained to recognize complex patterns in data sets. They have an advantage over conventional statistical methods in that they are not constrained by predefined mathematical relationships between dependent and independent variables and are thus able to model complex non-linear parameters. In recent decades, ANNs have become widely adopted in vari- 
ous industries due to their ability to solve tasks that are hard or impossible to solve using other conventional methods. At the same time, several urologic applications for ANNs have also been reported. Particular research has focused on several subspecialty areas, including male infertility, ${ }^{6}$ uro-oncology, 7,8 endourology ${ }^{9}$ and pediatric urology. ${ }^{10}$

In this study, a neural network-based system has been developed specifically to help in the management of antenatally diagnosed UPJ obstruction. For this purpose, a neural network-based prediction model was created and compared with multiple linear regression.

\section{Methods}

Between 2004 and 2008, 53 infants with antenatally detected hydronephrosis caused by UPJ obstruction were followed. All patients with antenatal hydronephrosis were evaluated with ultrasound examination after 48 to 72 hours. A voiding cystourethrogram was performed on all infants with hydronephrosis to exclude vesicoureteral reflux. Patients with vesicoureteral reflux, posterior urethral valve, ureterocele and obstructive megaureter were excluded from this study. We carried out a diuretic renogram and a dimercaptosuccinic acid (DMSA) scan in all patients at the age of 1 month. We performed dismembered pyeloplasty when significant hydronephrosis (renal pelvic diameter $>50 \mathrm{~mm}$ ) was noted on the sonogram or the renal scan showed $<40 \%$ of function in the affected kidney, especially if washout was delayed. If kidney function was $\geq 40 \%$, the patient was followed up with ultrasound and diuretic renography. Renal ultrasound examinations were performed every 3 months until the child reached 1 year of age, every 6 months for the next 2 years and then annually. If the renal sonogram showed increasing hydronephrosis, diuretic renography was repeated to confirm that renal function was stable. If renography showed deterioration of $>10 \%$ on the affected side, surgery was performed. These studies were undertaken more frequently (every 3 to 4 months) if renal function was borderline and significant hydronephrosis was present. At the end of the follow-up period, the patients' parameters were recorded in a commercially available software (Alyuda Research, Inc., Los Altos, CA), and a decision-aid model was developed. These data were also entered into a statistical software package (SPSS for Windows 12.0, IBM SPSS, Inc, Somers, NY) and linear regression analysis was carried out.

\section{Neural network}

As a part of this process, input neurons (numeric or categorical data which effect output) were created for every input variable (e.g., age, sex, split renal function) and an output neuron (resulting data) was created for the output variable (observation and surgery).
The network, which best describes the relationship between input and output variables, was conducted for the best feed-forward neural network architecture with a supervising learning algorithm (batch back-propagation). The search for the best network architecture was made in order to fine-tune the inputs. For example, for the network architecture of separated renal function, the least number of misclassifications was obtained for the network with 11 neurons in input layer, 4 neurons in a single hidden layer and 1 neuron in output layer. Therefore, this network structure was chosen for further consideration. The network accepted 8 inputs and 1 output trained for values (surgery or observation). The patients' age, sex, renal pelvic diameter, laterality, separated renal function on radionuclide scan, presence of renal scar on DMSA scan, urine culture results and the presence of symptomatic infections were used as input variables (Fig. 1).

Tenfold cross validation (random testing on training data) was used to test the accuracy of the classification. A 10-fold system (10 times system should run) was performed which split the data between training and testing cases. After this is complete, the average error rate is computed.

Of the 53 infants with UPJ obstruction, 37 (69.8\%) were randomized in the training sets. Eight children were randomized as the test sets and the remaining 8 children were randomized as the validation sets. The training set was used to train the neural network, a procedure that reduces the least square error between the correct response and the actual response until there is an acceptable overall error. New

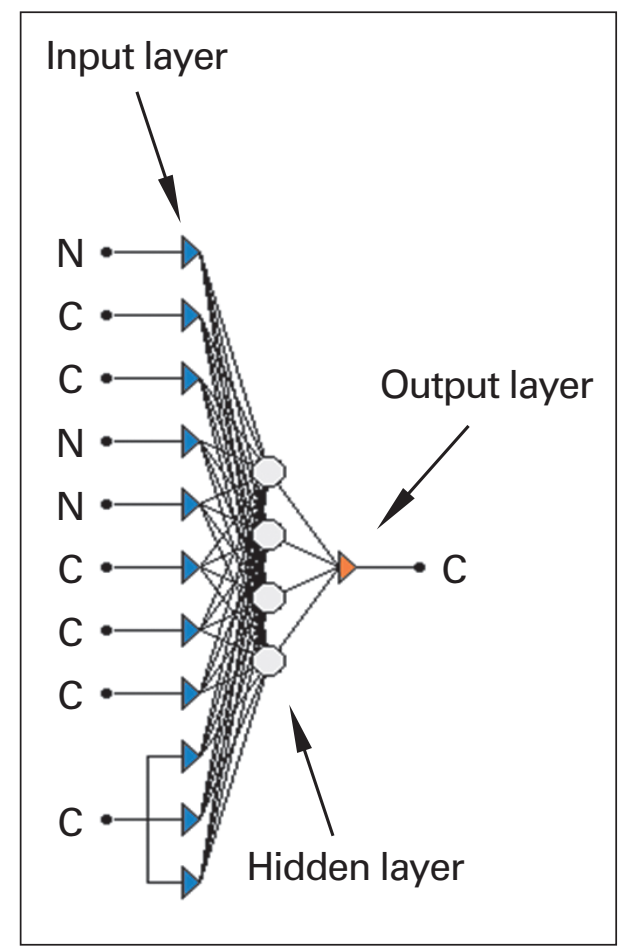

Fig. 1. Architecture of the current algorithm. 
Seçkiner et al.

\begin{tabular}{lc}
\hline Table 1. Patient characteristics & \\
\hline Average age (months) & $22.8 \pm 10.9$ (range 2-62) \\
\hline Sex $(\mathrm{n})$ & \\
Male & 40 \\
Female & 13 \\
Renal pelvic diameter (mean $\pm \mathrm{SD}$ ) & $29.1 \pm 12.9$ \\
Laterality & 34 \\
Left & 19 \\
Right & $10(19)$ \\
Positive urine culture, $\mathrm{n}(\%)$ & $5(9)$ \\
Symptomatic urinary infection, $\mathrm{n}(\%)$ &
\end{tabular}

SD: standard deviation.

data to which the system had not been exposed were then presented to the network; the network's performance was then evaluated.

Surgery and observation composed the prediction variables. These variables were used only as a teacher's response during the network's training with the batch back-propagation algorithm. The batch back-propagation algorithm is the most popular algorithm to train multi-layer perceptions. The convergence behaviour of the back-propagation algorithm depends on the learning rate and momentum parameters. The learning rate and the momentum used in training were 0.1 for the output variable.

\section{Results}

The mean follow-up time was 36.2 months. During the follow-up period, 36 children (68\%) were observed and the remaining 17 children (32\%) underwent pyeloplasty (Table 1 ). The average sensitivity of the ANN model in predicting the outcome was $92 \%$ in the training groups $(n=37)$ and $75 \%$ in the validation and test groups $(n=16)$. The specificity and the positive and negative predictive values in the training group were $77 \%, 88 \%$ and $83 \%$, respectively. After the training process, the neural network reported the importance of each predictor as follows (from most to least important): $69.6 \%$ for age, $16.4 \%$ for split renal function on diethylene triamine pentaacetic acid (DTPA), $6.1 \%$ for renal pelvic diameter, $0.5 \%$ for symptomatic urinary infection, $0.2 \%$ for laterality, $0.2 \%$ for urine culture results, and $0.03 \%$ for gender. In linear regression, none of the predictors were statistically significant (Table 2).

\section{Discussion}

Humans have a limited ability to evaluate and discriminate complex models, especially multidimensional relationships among variables. It is often difficult or impossible to discriminate between dependent and independent variables (i.e., age, comorbidity, prevalence of the disease and clini-

\begin{tabular}{lcc}
\hline \multicolumn{3}{l}{ Table 2. Correlation coefficients of the predictors in linear } \\
regression model & $\begin{array}{l}\text { Correlation } \\
\text { coefficients }\end{array}$ & p value \\
\hline Predictors & -0.196 & 0.239 \\
& 0.084 & 0.591 \\
Age & 0.124 & 0.397 \\
Sex & -0.219 & 0.181 \\
Laterality & 0.108 & 0.496 \\
Renal pelvic diameter & 0.021 & 0.902 \\
Split renal function & 0.063 & 0.717 \\
Symptomatic infection & & \\
Positive urine culture & &
\end{tabular}

cal and biochemical data). An ANN can correlate different predicting factors, find hidden interactions among variables, predict an outcome for a patient or groups, classify patients in risk groups or approximate a function and complete a known pattern. Most of the published papers on ANN in urology have demonstrated similar or better performance with ANN than with multivariate analysis, and a significantly improved accuracy than with univariate analysis. ${ }^{7,10-15}$ In addition, many researchers agree that the use of information technology, such as ANNs, is unavoidable in health care system management. ${ }^{16}$ This trend has triggered the application of the neural network approach to this problem. To the best of our knowledge, this paper describes the first neural network-based approach to predict treatment outcome of UPJ obstruction.

Today, there is a lack of consensus among pediatric urologists over the management of patients with UPJ obstruction. The key questions are who should be operated on and when. The reason for this lack of consistency is the absence of an accurate diagnostic tool to differentiate obstructive from nonobstructive hydronephrosis. A number of diagnostic studies, such as ultrasonography, radionuclide renography, magnetic resonance imaging and pressure flow studies, have been and are still used to define whether a kidney is obstructed. Diuretic renography is considered the radiographic study that best defines the presence of a UPJ obstruction.

In the present ANN algorithm, supervised learning has been applied to solve this problem. Supervised learning is based on an "external teacher" that tells each output unit (nominal variable) what its desired input signals should be and/or the difference in the outputs (continuous variable). Thus, ANN changes the weights according to the learning rule. In addition, we used a batch back-propagation learning algorithm, the most popular algorithm to train multi-layer perceptrons; this algorithm is often used by researchers and practitioners.

Our ANN model has produced varying prediction accuracies depending on the treatment variables and the accuracy of the historical data used. We found the average success rate of the ANN model in predicting outcome was $92 \%$ in the training groups and $75 \%$ in the validation and test groups. 
Neural networks in ureteropelvic junction obstruction

Artificial neural networks have been shown to yield superior predictive capability compared with the multiple linear regression model.

There are some limitations to this approach. Firstly, data sets should be updated at least every 2 to 3 years. Secondly, if the network is not sufficiently trained, outputs are not reliable for clinical use. Pediatric urologists can easily enter their cohorts' data to personalize the network. Once the training and validation of the network are completed, new patient data can be entered to define the treatment. It is difficult to predict whether this tool could replace clinical judgment, however, we believe that it would be a valuable addition for busy pediatric urologists.

\section{Conclusion}

We have demonstrated that the use of ANNs in antenatally diagnosed UPJ obstruction can help the clinician in making treatment decisions and can be useful in daily clinical practice. Moreover, ANNs may have a role in complementing traditional methods to produce a more accurate prediction of clinical outcome.

*Department of Urology, University of Gaziantep, Gaziantep, Turkey; ${ }^{\dagger}$ Department of Industrial Engineering, University of Gaziantep, Gaziantep, Turkey

Competing interests: None declared.

This paper has been peer-reviewed.

\section{References}

1. Lee RS, Cendron M, Kinnamon DD, et al. Antenatal hydronephrosis as a predictor of postnatal outcome: a meta-analysis. Pediatrics 2006; 118:586-93.

2. Estrada Cr Jr. Prenatal hydronephrosis: early evaluation. Curr Opin Urol 2008;18:401-3.

3. Koff SA. Postnatal management of antenatal hydronephrosis using an observational approach. Urology 2000;55:609-11.

4. Josephson $\mathrm{S}$. Antenatally detected pelvi-ureteric junction obstruction: concerns about conservative management. BJU Int 2000;85:973.

5. Sutherland RW, Chung SK, Roth DR, et al. Pediatric pyeloplasty: outcome analysis based on patient age and surgical technique. Urology 1997;50:963-6.

6. Samli MM, Dogan I. An artificial neural network for predicting the presence of spermatozoa in the testes of men with nonobstructive azoospermia. J Urol 2004;171:2354-7.

7. Stephan C, Meyer HA, Kwiatkowski M, et al. A $(-5,-7)$ proPSA based arrificicial neural network to detect prostate cancer. Eur Urol 2006;50:1014-20.

8. Borque $A$, Sanz $G$, Allepuz $C$, et al. The use of neural networks and logistic regression analysis for predicting pathological stage in men undergoing radical prostatectomy: a population based study. I Urol 2001;166:1672-8.

9. Parekattil SJ, Kumar U, Hegarty NJ, et al. External validation of outcome prediction model for ureteral/ renal calculi. J Urol 2006;175:575-9.

10. Seckiner I, Seckiner SU, Erturhan $S$, et al. The use of artificial neural networks in decision support in vesicoureteral reflux treatment. Urol Int 2008;80:283-6.

11. Crawford ED. Use of algorithms as determinants for individual patient decision making: National comprehensive cancer network versus artificial neural networks. Urology 2003;62 (Suppl 6A):13-9.

12. Remzi M, Anagnostou T, Ravery V, et al. An artificial neural network to predict the outcome of repeat prostate biopsies. Urology 2003;62:456-60.

13. Zlotta AR, Remzi M, Snow PB, et al. An artificial neural network for prostate cancer staging when serum prostate specific antigen is $10 \mathrm{ng} / \mathrm{ml}$ or less. J Urol 2003; 169:1724-8.

14. Remzi M, Djavan B. Arrificial neural networks in Urology 2004. Eur Urol Supplements 2004;3:33-8.

15. Serrano-Durba A, Serrano AJ, Magdalena JR, et al. The use of neural networks for predicting the result of endoscopic treatment for vesico-ureteric reflux. BJU Int 2004;94:120-2.

16. Ozcan YA. Quantitative Methods in Health Care Management: Techniques and Applications. San Francisco, CA, Jossey-Bass/Wiley, 2005

Correspondence: Dr. Ilker Seçkiner, Associate Professor of Urology, University of Gaziantep, School of Medicine, Department of Urology, 27310 Gaziantep/Turkey; fax: +90 342 3603998; iseckiner@yahoo.com 